Symmetries, Differential Equations and Applications (SDEA-II)

International Journal of Modern Physics: Conference Series

Vol. 38 (2015) 1560070 (23 pages)

(C) The Authors

DOI: $10.1142 / \mathrm{S} 2010194515600708$

\title{
Sesquicentennial of the presentation by James Clerk Maxwell of his paper "A Dynamical Theory of the Electromagnetic Field" to the Royal Society of London
}

\author{
Asghar Qadir \\ School of Natural Sciences \\ National University of Sciences and Technology \\ Campus H-12, Islamabad 44000, Pakistan \\ asgharqadir46@gmail.com \\ D. P. Mason \\ DST-NRF Centre of Excellence in Mathematical and Physical Sciences \\ Differential Equations, Continuum Mechanics and Applications \\ School of Computational and Applied Mathematics \\ University of the Witwatersrand, Johannesburg \\ Private Bag 3, Wits 2050, South Africa \\ david.mason@wits.ac.za \\ Published 30 April 2015 \\ Let us work while it is day, for the night is coming and work by day \\ leads to rest by night
}

JC Maxwell

\begin{abstract}
James Clerk Maxwell is generally regarded as the greatest contributor to the development of Physics in the time between Newton and Einstein. His most important contributions are the Kinetic Theory of Gases and Electromagnetism which is the unified theory of Electricity and Magnetism. Although his major work on Electromagnetism was published in 1865 it was read at a meeting of the Royal Society of London in 1864. The sesquicentennial of the theory correctly falls in 2014. In this article that event is celebrated. Parts of his early and professional life are described. Aspects of his many contributions are discussed but mainly we concentrate on his contributions through thermal and electromagnetic Physics.
\end{abstract}

This is an Open Access article published by World Scientific Publishing Company. It is distributed under the terms of the Creative Commons Attribution 3.0 (CC-BY) License. Further distribution of this work is permitted, provided the original work is properly cited. 


\section{Introduction}

James Clerk Maxwell is generally regarded as the leading mathematical physicist of the era between Newton and Einstein. He was an outstanding applied mathematician with powerful physical insight. He introduced the important concept of the field into theoretical physics. He recognised the need to check his theoretical predictions experimentally and performed the experiments himself.

On 8th December 1864 Maxwell read before the Royal Society of London his paper "A Dynamical Theory of the Electromagnetic Field". The year 2014 marks the sesquicentennial of that presentation which is regarded as one of the most important events of classical physics. In this article we celebrate that presentation. The paper was later published in the Philosophical Transactions of the Royal Society in $1865 .^{1}$

We will describe parts of Maxwell's life and work. Of his many contributions to science, we will mention his work on colour and Saturn's rings and the establishment of the Cavendish Laboratory at the University of Cambridge, but we will mainly concentrate on his contributions to the Kinetic Theory of Gases for which he introduced statistical methods into Physics and to electromagnetism and the electromagnetic theory of light. There are many books on Maxwell. The texts which were consulted during the preparation of this paper are. ${ }^{2-6}$

\section{Life and work}

James Clerk Maxwell was born on 13th June 1831 in a three storey house at 14 India Street, Edinburgh. On his father's side he was descended from the Clerks of Penicuik in Midlothian, which was a distinguished Scottish family consisting of Parliamentarians and lawyers. Maxwell's great-grandfather had married his cousin Dorothea Maxwell and inherited the Maxwell estates provided he assume the Maxwell name, which he did as Clerk Maxwell. It was through his great-grandfather that Maxwell inherited the estate of Glenlair in Kirkcudbrightshire in south-west Scotland. His mother was Frances Cay from Northumberland. The Cay family had achieved distinction in the legal and mathematical fields. A member of the Cay family was Second in the Tripos at Cambridge University in 1752 and a Cay cousin was 6th Wrangler in 1864.

Maxwell spent his childhood on the Glenlair estate. His father John had designed and built the house on the estate and Maxwell in turn would add to the building. As a child Maxwell showed a curiosity of the world around him and wanted to know how it worked. If anything moved or shone he would ask the question, "what is the go of that?" His mother looked after his early education until she died from abdominal cancer in 1839 at the age of 47 years. For a time Maxwell was taught by a private tutor before starting school at Edinburgh Academy in 1841.

Maxwell spent six years at Edinburgh Academy. He received a good grounding in Latin and Greek and a good background in Mathematics and some Physics. Maxwell had a natural ability at geometry which he demonstrated from an early age. While still at school, in 1846, he published his first paper which was on oval curves. ${ }^{7}$ 
This geometrical vision contributed significantly to Maxwell's physical insight by allowing him to visualise bodies and their motion. Maxwell made friends with Peter Guthrie Tait who although the same age as Maxwell was a year behind him at school. The two remained friends throughout Maxwell's life. Tait was Senior Wrangler and First Smith's Prizeman at Cambridge University in 1852 and later became Professor of Natural Philosophy a at Edinburgh University. In their correspondence Maxwell was $d p / d t$ because of the equation $d p / d t=J C M$ in Tait's book on thermodynamics. Tait was $\mathrm{T}^{\prime}$ and William Thomson, Lord Kelvin, who became a friend of both Maxwell and Tait, was T.

Maxwell spent three years at Edinburgh University. He studied Logic, Physics, Mathematics, Chemistry and some Geology. The Professor of Natural Philosophy was James Forbes who had read Maxwell's paper on Oval Curves to the Royal Society of Edinburgh because Maxwell was considered too young to present the paper himself. Forbes allowed Maxwell to use his laboratory to perform his own experimental investigations. This was an important contribution to Maxwell's early scientific training in performing experiments. Forbes also helped Maxwell to develop a literary style for his papers which had authority but was still fresh and informal. ${ }^{6}$

Maxwell published two papers while at Edinburgh University. They were read to the Royal Society of Edinburgh by the Professor of Mathematics, Phillip Kelland, because Maxwell was still considered too young to present them himself. The first paper was a geometrical paper on Rolling Curves $^{8}$ which followed on from his work on Oval Curves. The second paper was on the Equilibrium of Elastic Bodies. ${ }^{9}$ It dealt with the deformation of solid bodies when subjected to stress. He developed the mathematical theory of photoelasticity based on elastic strain functions and verified the results with his own experiments which used polarised light. The field equations of elasticity had been developed by GG Stokes and in this study Maxwell acquired insight into field theory which he was to use later when setting out the equations of Electromagnetism. Maxwell used one of the colourful patterns from photoelasticity as a design for a woolen kettle holder which he hung from his fireplace at Glenlair. ${ }^{3}$

He went to Cambridge University in October 1850. He was in residence at Peterhouse for the Michaelmas term ${ }^{\mathrm{b}}$ and then migrated in December to Trinity College. He surprised the tutor (later Master of Trinity College) to whom he applied to migrate by producing a bundle of research papers and saying "Perhaps these may show you that I am not unfit to enter your College". ${ }^{2}$ His tutor was William Hopkins of Peterhouse who had prepared many Senior Wranglers for the Mathematical Tripos. Hopkins was methodical and brought order to Maxwell's work although Maxwell still took his own way in his studies. In 1853 Hopkins described Maxwell as

\footnotetext{
a At the time of Newton, there was no separate 'Physics', it was called 'Natural Philosophy'. This practice has been carried on in the older Scotish Universities.

b The Feast of Saint Michael is on 29th September, the beginning of the autumn (fall) term in a number of English-speaking universities.
} 
"the most extraordinary man he had met within the whole range of his experience; it appeared impossible for Maxwell to think incorrectly on physical subjects ${ }^{10 "}$. If the subject allowed, Maxwell used diagrams although the other students may have solved the problem more easily using analysis. He proceeded from one idea to another instead of relying on mathematical symbols and equations. He was a member of the Apostle Club and the essays he prepared contributed to the high literacy quality of his later papers. He attended regularly the lectures of George Gabriel Stokes. Maxwell was already acquainted with some of the papers of Stokes from his own work on elasticity. His friend Tait has observed that "it may safely be said that no high Wrangler of recent years ever entered the Senate-house more imperfectly trained to produce 'paying' work than did Clerk Maxwell ${ }^{10 "}$. Maxwell was Second Wrangler in the Mathematical Tripos. The Senior Wrangler was the Canadian Routh. He was first equal with Routh in the Smith's prize which had a separate examination.

In 1854 Maxwell started postgraduate research at Cambridge University. He was elected a Fellow of Trinity College in October 1855. He worked in two main areas, colour vision and Faraday's lines of force although he also started research in the kinetic theory of gases which became an interest for the rest of his life. In March 1855 he read to the Royal Society of Edinburgh a paper on the mixture of colours and colour blindness. ${ }^{11}$ It was mainly an experimental paper. By using multicoloured spinning discs invented by Newton and developed by Forbes at Edinburgh University he showed how any colour could be represented by a suitable mixture of three primary colours, red, green and blue. Thomas Young, fifty years earlier, had taken the primary colours to be red, green and violet. Maxwell described the rules of colour combination with the aid of an equilateral colour triangle with red, green and blue at each vertex. White light is obtained from an equal mixture of red, green and blue. With paints, an equal mixture of red, yellow and blue pigments gives white but Maxwell found that no mixture of red, yellow and blue light gave white light. There is an essential difference between mixing pigments as with paints and mixing lights. The pigments extract colour so that the light that is seen is the colour the paint was unable to absorb while mixing lights is combining colours. ${ }^{6}$ It is relevant to remark that the Nobel Prize for Physics 2014 was awarded jointly to Isamu Akasaki, Hiroshi Amano and Shuji Nakamura "for the invention of efficient blue light-emitting diodes which has enabled bright and energy-saving white light sources". $\mathrm{In}^{12}$ it is stated that: "A little further on in the future three-colour light-emitting diodes may replace the combination of blue light emitting diodes and phosphor for efficient lighting". Maxwell tiled the floor of the entrance hall to his house at Glenlair with coloured tiles as shown in Fig. 1. Red, green, blue and white tiles feature prominently in the floor pattern.

He read a paper $^{13}$ on Faraday's lines of force to the Cambridge Philosophical Society in two parts, the first on 10 December 1855 and the second on 11 February 1856. It formulated mathematically Faraday's idea of a line of force and replaced the model of action-at-distance by the concept of a field. It was the second 


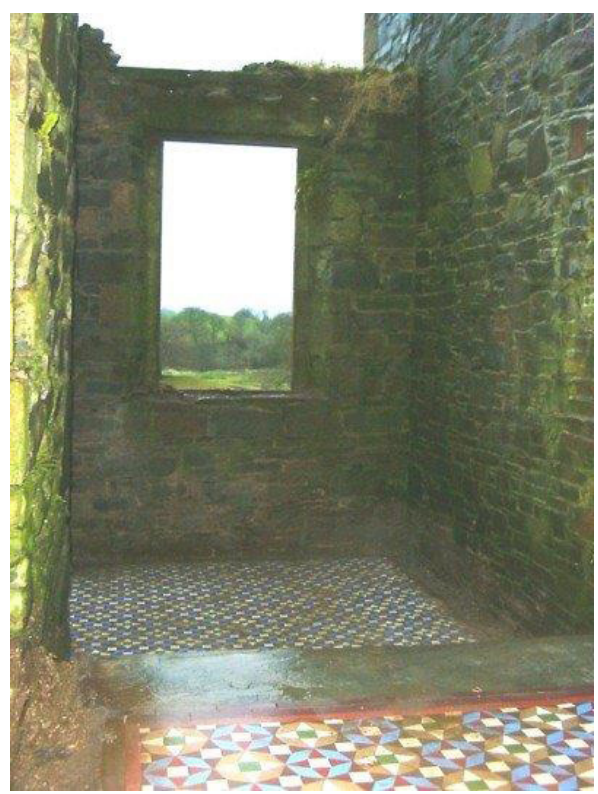

Fig. 1. Tiles laid by Maxwell on the floor of the entrance hall to Glenlair.

longest of Maxwell's research papers and marked the beginning of his work on Electromagnetism.

The Chair of Natural Philosophy (Physics) in Marischal College, Aberdeen, became vacant in 1855 and he applied. He would be closer to Glenlair and to his father who was not in good health and he would also have a laboratory. He was appointed to the Chair but his father died in April 1856. He resigned as a Fellow of Trinity College, Cambridge but was later elected an Honorary Fellow. He started his duties at Marischal College towards the end of 1856.He gave fifteen lectures a week and also evening classes for artisans, a practice he started at Cambridge and was to continue while at King's College, London. He introduced the idea of students doing experiments themselves instead of only watching them being done. He was to do this also at London University and at the Cavendish Laboratory in Cambridge. One of his students was George Reith who was father of John Reith, later Lord Reith, the first Director-General of the BBC. Maxwell signed the class certificate of George Reith which Lord Reith hung in his office at the BBC. This linked Maxwell who discovered electromagnetic waves with Reith who shaped their application in broadcasting. ${ }^{4}$ In 1858 Maxwell married Katherine Dewar, the daughter of the Principal of Marischal College.

Maxwell continued his research on colour vision at Aberdeen. A long paper on the theory of compound colours was published by the Royal Society of London which describes observations by himself and his wife Katherine to determine the 
spectral curve on the colour triangle. ${ }^{14}$ In 1860 he was awarded the Rumford Medal of the Royal Society of London for his work on colour vision. Maxwell made two other significant research contributions at Aberdeen, his essay for the Adams Prize and his work on the Kinetic Theory of Gases.

The Adams Prize Essay had been founded in 1848 by St John's College, Cambridge, to commemorate the theoretical discovery of the planet Neptune by John Couch Adams. In 1843 Adams was Senior Wrangler and also won the First Smith's prize. ${ }^{15}$ The Prize was set every two years and open to Cambridge graduates. The subject in 1855 was to investigate the structure of Saturn's rings. Maxwell put a lot of effort into this investigation. It was already known that a ring could not be a uniform solid because it would be unstable. Maxwell showed that a ring could be a nonuniform solid body but the required distribution of mass did not agree with observations. He also showed that unstable waves would be formed in a fluid ring. He therefore concluded that a ring could only be composed of an indefinite number of disconnected masses. The masses revolved round Saturn with different velocities according to their distances from the planet. In December 1856 Maxwell submitted the essay "On the stability of motion of Saturn's rings", which was later published. ${ }^{16}$ It was over 80 pages in length and full of mathematical techniques and physical insight. Due to the difficulty of the subject it was the only entry. The Astronomer Royal, Sir George Airy, described it as one of the most remarkable applications of Mathematics to Physics that he had ever seen. Maxwell was awarded the Adams Prize for 1855. His prediction was confirmed by the photographs sent back to Earth by the Voyager 1 and 2 spacecraft in the early 1980s.

Maxwell was attracted to the Kinetic Theory of gases by the work of Rudolf Clausius who was developing a molecular theory of gases. It has also been suggested that his model of Saturn's rings as made up of a very large number of masses with a range of velocities depending on distance from the planet led him into Kinetic Theory. His first contribution was the Maxwell distribution law giving the velocity distribution of molecules in thermal equilibrium in a gas. He presented the law at the meeting of the British Association for the Advancement of Science in Aberdeen in September 1859. The derivation of the Maxwell distribution law made no reference to collisions between molecules. It may be the first law of physics that depends on probability. The probabilistic model of matter is now fundamental to physics. The presentation was published as a paper in two parts in $1860 .{ }^{17}$ The paper covered a wide range of topics in Kinetic Theory and is regarded as one of Maxwell's greatest papers. It contained the counter intuitive prediction that the viscosity of a gas is independent of its pressure. This was verified in 1865 by experiments which Maxwell and his wife, Katherine, performed in London.

In 1860 the two colleges in Aberdeen, Marischal College and King's College, were fused together to form Aberdeen University. The new Chair of Natural Philosophy was filled by the former professor at King's College who was senior to Maxwell. Maxwell's post ceased to exist and he had to find another position. Both Maxwell and Tait applied for the Chair of Natural Philosophy at Edinburgh University 
which became vacant on the retirement of Forbes. Tait was appointed to the Chair. Maxwell then applied for the vacant Chair of Physics and Astronomy at Kings College, London and was accepted in July 1860. Before traveling to London, Maxwell spent the summer at Glenlair. He contracted smallpox, possibly when he went to buy a horse at a local fair. He was very ill but Katherine took great care of him. In later life Maxwell said that she saved his life.

Maxwell was Professor at King's College from 1860 to 1865. It was full of scientific activity and the most creative time of his career in which his most important papers were published. They moved into a large house of four storeys at 8 (now 16) Palace Gardens Terrace, Kensington. It was 4 miles to King's College which he could walk or take a horse-drawn bus ride. He was able to walk to meetings of the Royal Society and at the Royal Institution. Kensington Gardens and Hyde Park were close by where Maxwell and Katherine, who were enthusiastic horse riders, frequently went for a ride in the afternoon. The house also had a large attic where Maxwell did experiments with the assistance of Katherine. A lecturer was appointed to assist Maxwell in his duties. The post was eventually held by Grylls Adams who was the younger brother of John Adams in whose honour the Adams Prize was founded. Adams succeeded Maxwell to the Professorship when he resigned in 1865 . In his inaugural lecture Maxwell stressed that he wanted above all to help people learn to think for themselves.

While Maxwell was at Cambridge he had started corresponding with Faraday. He now met him personally. He would often attend sessions at the Royal Institution including Faraday's Friday evening lectures. They got along well based on a strong mutual respect but the relation was not close because Faraday was 40 years older than Maxwell. Maxwell and Faraday had dinner together in May 1861 when Maxwell gave a lecture at the Royal Institution on colour vision. At this lecture Maxwell produced the first colour photograph which was of a tartan ribbon. He took black and white photographs through red, green and blue filters and then projected and superposed the results through the same filters. ${ }^{3}$ Three weeks after his lecture at the Royal Institution he was elected a Fellow of the Royal Society of London in recognition of his research on Saturn's rings and colour vision. The following week he celebrated his 30th birthday.

Maxwell, with the assistance of Katherine, performed experiments to test his prediction from Kinetic Theory that the viscosity of a gas is independent of pressure. The gas used was air. He determined the viscosity by measuring the rate of damping of the oscillation of a stack of three discs suspended by a thin wire. This torsional pendulum was sealed inside a large glass vessel and the air pressure was adjusted by a pump. He also tested experimentally a second prediction that the viscosity should vary as the square root of the absolute temperature. Maxwell analysed the two sets of results at Glenlair during the summer break. Calculations then were done using log tables. He forgot to take his log tables with him and he had to do all the calculations by hand. ${ }^{6}$ The experimental results showed that the viscosity was constant over a wide range of pressure which verified his prediction. The second set 
of experimental results showed that the viscosity did not vary as the square root of the absolute temperature but came closer to varying as the absolute temperature. The two laws were derived from Kinetic Theory under different assumptions. The pressure dependence law held for any type of molecule while the temperature law depended on the assumption that the molecules were perfectly elastic spheres colliding with each other. Also Clausius had found errors in Maxwell's derivation of the law of equipartition of energy that the kinetic energy in a gas is divided equally between linear and rotational energy. These and other considerations motivated Maxwell to undertake further investigations in Kinetic Theory which led to his major paper "On the dynamical theory of gases". ${ }^{18}$

Maxwell was a member of the committee of the British Association for the Advancement of Science which was formed to investigate a system of units for electricity and magnetism. Experiments were performed at King's college. Maxwell introduced the method of dimensional analysis in which all physical quantities are defined in terms of mass, length and time. The recommendations of the Committee made a significant contribution to the international system of units adopted in 1881 .

Maxwell's most important work at King's College was on Electromagnetism. In paper $^{13}$ he introduced the displacement current and showed that light waves were electromagnetic waves. Polarised light had been an interest of Maxwell since, as a school boy at Edinburgh Academy, he had been taken to the workshop of the optician William Nicol who had discovered how to polarise light using prisms cut from Iceland spar. In paper ${ }^{1}$ which we are celebrating he presented the equations of Electromagnetism, now known as Maxwell's equations and was able to explain polarization. His prediction of electromagnetic waves was eventually confirmed experimentally by Heinrich Hertz in 1888. The implications of this will be discussed in more detail in Sec. 4.

Maxwell resigned from King's College in January 1865 and a few months later returned to Glenlair which was to be his main residence until 1871 . He wanted to have time to write-up his research and also to improve his estate. He enlarged the house on the estate during 1867. A bridge was built across the river Urr by his cousin William Cay who was a Civil Engineer. Figure 2 contains a letter written by Maxwell a little later in 1876 which illustrates the commitment he made to the running of his estate. He visited his tenants and took an interest in the local school. Life in the country may have inspired the paper "On hills and dales" which was an early exercise in topology. ${ }^{19}$ He maintained a correspondence with his colleagues, especially Stokes, Tait and Thomson (Lord Kelvin) and received journals and proofs of his manuscripts by mail. He had a special post box built in a stone wall about $1 / 2$ mile from the house and would walk there and back each day to take and collect mail. He spent the winters in London and was an external examiner for the Mathematical Tripos at Cambridge.

Maxwell continued his research on the dependence of the viscosity of a gas on temperature. Instead of modelling the gas molecules as colliding perfectly elastic spheres, he assumed that they repel each other by a force that is proportional to 


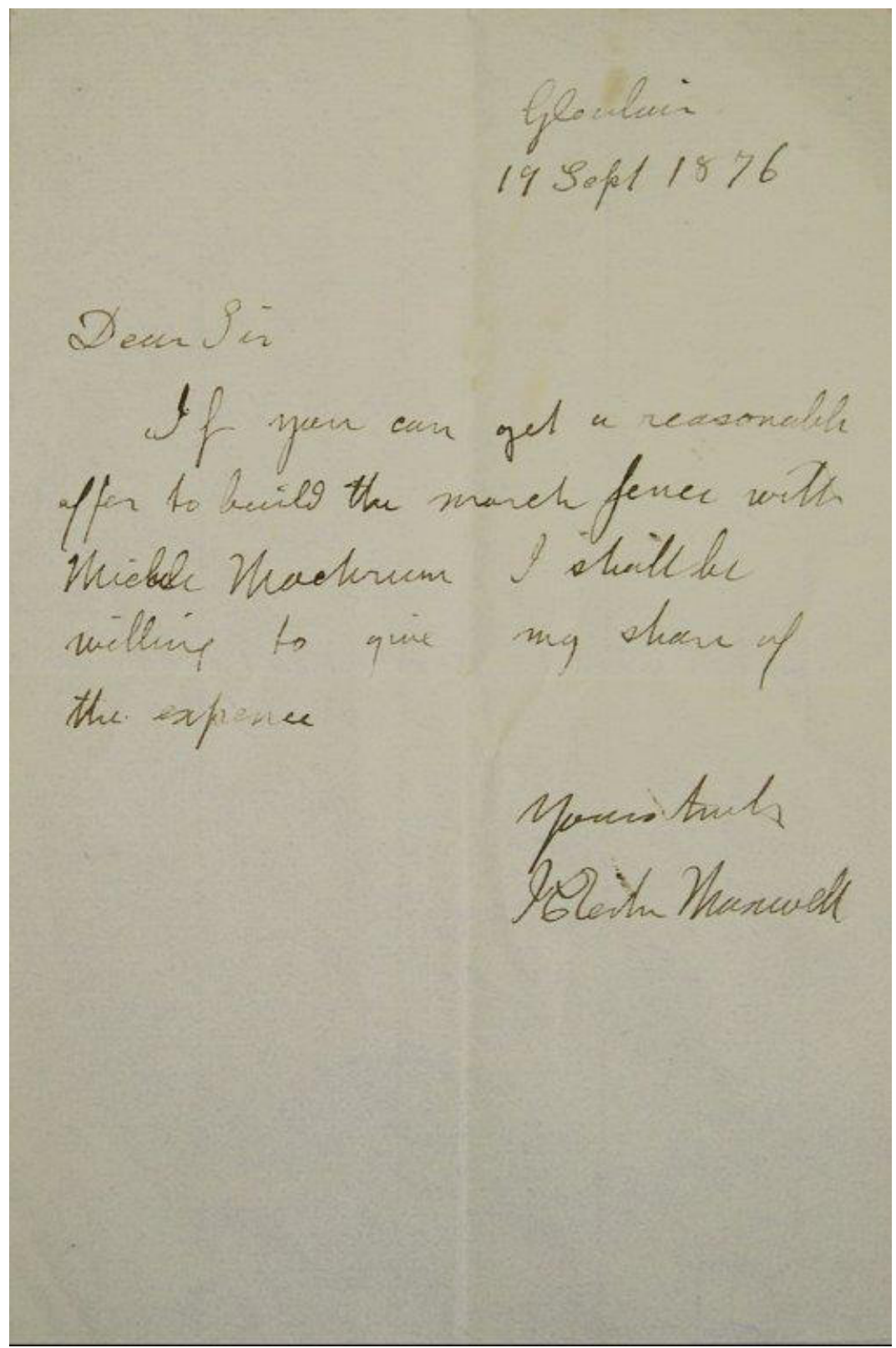

Fig. 2. Letter written by Maxwell on improvement to his Glenlair estate.

$r^{-n}$ where $r$ is the separation distance. He found that the mathematics could be greatly simplified by setting $n=5$ and that this also gave a linear relation between viscosity and absolute temperature. This agreed better with his experiments in London but further experiments by other investigators showed that in general the 
viscosity was not proportion to the temperature although for some gases $n=5$. In his derivation of relations for viscosity, diffusion and thermal conductivity he introduced the concept of a relaxation time which is now widely used in science. His paper "On the dynamical theory of gases" 18 in which this work was published gave strong support to the model that gases are composed of molecules.

Maxwell wrote the book "The theory of heat" while at Glenlair. ${ }^{20}$ It contained for the first time Maxwell's thermodynamic relations between pressure, volume, temperature and entropy. His contribution in this regard will be discussed later in Sec. 3 .

The main part of Maxwell's greatest work, "Treatise on electricity and magnetism" was written at Glenlair between 1865 and 1871. A photograph of the bay window of the room in which he wrote the Treatise is shown in Fig. 3. It was published in 1873 after Maxwell had returned to Cambridge. The writing of the Treatise gave him the opportunity to organise his own thoughts on the subject. It contained almost everything then known on electricity and magnetism. It also contained new results on the radiation pressure exerted by electromagnetic waves including light. Maxwell used two representations for the equations, the cartesian component form and the more concise quaternion form. He introduced the terminology, curl, gradient and convergence to help with the physical interpretation. The terms curl and gradient are now used in vector calculus but convergence has been changed to its negative, divergence. The 1000 pages of the Treatise contain the word "aether" only once. ${ }^{3}$

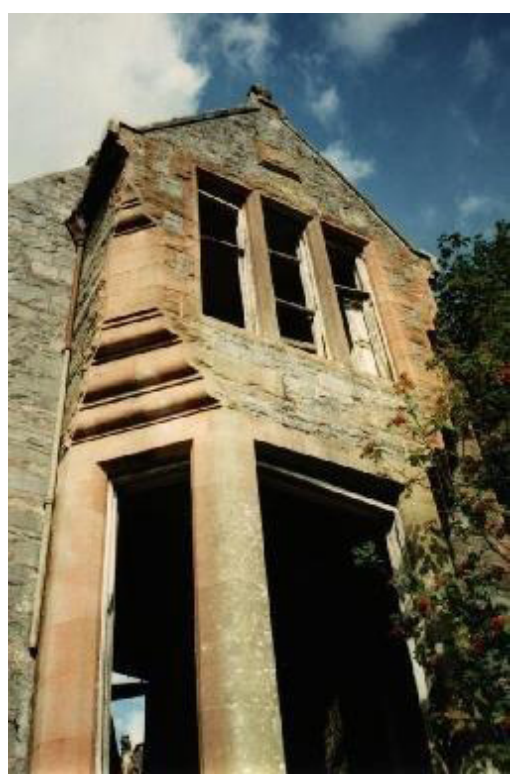

Fig. 3. The bay window of the room at Glenlair in which Maxwell wrote his Treatise on Electricity and Magnetism. The house was largely destroyed by fire in 1929 . 
Maxwell wanted to concentrate on the consequences of his theory rather than on its foundations. The Treatise ranks after Newton's Principia as the most important book in the history of physics. The Chair of Experimental Physics at Cambridge University was established in 1870. After Thomson and Helmhotz had declined the appointment it was offered to Maxwell who accepted on 8 March 1871. The Chancellor of the University 'The eighth Duke of Devonshire', William Cavendish, had donated funds to build a Physics Laboratory and to purchase equipment. Maxwell visited Thomson's Laboratory at Glasgow University to learn from their experience. He designed the building with the architect as he had done when extending Glenlair. He took great care to select the best equipment. He designed some of the apparatus himself. The building had been known as the Devonshire Laboratory. ${ }^{6}$ The name was changed to the Cavendish Laboratory at the suggestion of Maxwell to honour both the Duke and his great uncle, the physicist Henry Cavendish. The Cavendish Laboratory was completed in 1874 .

In his inaugural lecture Maxwell pointed out how "careful measurement has been rewarded by the discovery of new fields of research and by the development of new scientific ideas". As Director of the Cavendish Laboratory one of Maxwell's duties was to oversee the research in the Laboratory. He would suggest a research problem if asked but otherwise would let the students choose their own topic. Maxwell was an excellent supervisor of students in a laboratory and was greatly respected. Students were attracted to the Cavendish Laboratory including Strutt (Lord Rayleigh), Poynting and Fleming who invented the thermionic valve. There was a revival of Physical Sciences at Cambridge.

In 1874 Maxwell was asked by the Duke of Devonshire to edit for publication the papers of Henry Cavendish, who was more interested in doing research than in publishing. Maxwell was impressed by the originality of Cavendish's work. Many of the experiments were on electricity which Maxwell performed again. The editing took a lot of time but it was completed and a book was published in 1879. ${ }^{21}$

The most important paper Maxwell wrote while at the Cavendish Laboratory was inspired by Boltzmann's work on the Kinetic Theory of gases. ${ }^{22}$ Maxwell introduced the idea of an ensemble average and stated the ergodic hypothesis. He gave a new derivation of the distribution of velocities of a gas in equilibrium which led to a proof of the principle of the equipartition of energy. He also showed that mixtures of gases could be separated by a centrifuge. This paper is one of the foundations of statistical mechanics.

Maxwell's health had always been good until the beginning of 1877 when he developed heartburn. By April 1879 he found it difficult to swallow. He spent the summer at Glenlair. On 2 October he was told by his doctor that, like his mother, he had abdominal cancer and had about one month to live. He returned to Cambridge to have the assistance of Dr Paget and be with his friends and colleagues. He died on 5 November 1879. He is buried in Parton Churchyard near his Glenlair estate. The house at Glenlair was largely destroyed by fire in 1929 . 
Maxwell died at the height of his powers. The characteristics he demonstrated when young remained with him all his life. He had a large capacity for sustained work. He had great powers of concentration even when there were distractions, a retentive memory, a high degree of originality, a deep physical insight and mathematical ability. Although reserved he had confidence in his own powers as a scientist. He was kind and patient with a lively sense of humour which is clearly displayed in his poetry and correspondence. Maxwell was eccentric in his manners and he was sometimes difficult to follow in conversation. At meals he was often lost in thought. He prepared his lectures very conscientiously and thoroughly but they were not well attended. Horace Lamb who attended his lectures at Cambridge said that "he had his full share of misfortunes at the blackboard". David Gill, a student of Marschal College and future Director of the Royal Observtatory, Cape of Good Hope (in what is now South Africa), commented that "to those who could catch a few of the sparks that flashed as he thought aloud at the blackboard in lecture, or when he twinkled with wit or suggestion in after-lecture conversation, Maxwell was supreme as an inspiration" ${ }^{6}$ He demonstrated social responsibility and compassion throughout his life. He leant books from the library at Glenlair to young farm workers. When a youth he preferred to travel by third class in a railway journey. He said that he preferred a hard seat. At Cambridge, Aberdeen and London he gave evening courses at Working Men's colleges. As a research supervisor in the laboratory he inspired his students with his generous advice and kindness. He liked country life and was a good horseman. He was conscientious in his responsibilities to his tenants on the Glenlair estate. He was of average height, strong and sturdy from his country upbringing and walked with a spring in his step. At Cambridge he walked every day from his house to the Cavendish Laboratory with his dog Tobi. We have this word picture of Maxwell working in the Cavendish Laboratory: ${ }^{3}$

In performing his private experiments at the laboratory Maxwell was very neathanded and expeditious. When working thus, or when thinking about a problem, he had a habit of whistling, not loudly, but in a half-subdued manner. He could carry the full strength of his mental facilities rapidly from one subject to another and could pursue his studies under distractions which most students would find intolerable. On these occasions he used in a manner to take his dog into his confidence and would say softly, 'Tobi, Tobe', at intervals and after thinking and working for a time, would at last say "It must be so; Plato thou reasonest well" c.

\section{The Kinetic Theory of Gases}

Although Maxwell's greatest achievement is regarded as his theory of Electromagnetism, his contribution through his attempts to explain thermal phenomena has no less far-reaching consequences. It is worth while to review the understanding of heat and the thermal properties of matter prevalent at the time.

\footnotetext{
${ }^{\mathrm{c}}$ The verse is from Cato: A Tragedy (1713) by Joseph Addison (1672-1719).
} 
Aristotle had believed that there are four terrestrial "elements", viz. earth, water, air and fire; and a fifth celestial element, "aether", on the basis of the five Platonic solids (whose faces are regular polygons). Chemical studies in the mid 1660's did not fit in with this view of fire fully and the "phlogiston theory" of heat was proposed. It regarded heat as a "fifth" terrestrial element, violating the Aristotelian basis. Later chemical investigations demolished the five elements of Aristotle and introduced many more. Now a fluid theory of heat was proposed which did not fare much better. With the observation of the heating effect of mechanical work and the use of heat to do mechanical work, a much better understanding was required. This came with the First Law of Thermodynamics, which related heat to work and essentially gives the Law of Conservation of Energy.

With the concept of energy there was the need to provide an internal energy for any system. This energy could be due to mechanical work and would then be the product of the pressure and volume of a gas, or it could be chemical energy as when combustion occurred, or it could be due to sound, or it could be due to heat. Sound was understood as compression waves in the medium but heat remained to be understood. The intensity of heat being the temperature there was need for a quantity corresponding to the volume that, when multiplied by the temperature, would give the heat component of the internal energy. The problem was that there was no such quantity seen in the laboratory. This postulated quantity was labeled entropy. Investigations led to the statement of the Second Law of Thermodynamics that concerned this entropy. It was a very different kind of law from the laws of mechanics or the First Law of Thermodynamics; it was an inequality instead of being an equality! What it said was that the entropy of a closed thermodynamic system always increases. Since the efficiency of a heat engine reduces as entropy increases, this direful law seemed to say that the Universe will "wind down".

Before his major innovation, Maxwell had already made significant contributions to thermodynamics by the use of partial differential calculus to relate rates of change of different thermal quantities. Maxwell's relations led to clear physical implications of entropy that were observed. (They are still taught to Physics students, but the contribution could hardly be regarded as revolutionary.)

He now addressed the two major problems in thermodynamics: the nature of heat; and the concept of entropy and the Second Law of Thermodynamics. Mechanics, being regarded as a sound base, Clausius and others used Bernoulli's view of fluids as composed of molecules. They tried to explain heat as the kinetic energy of the molecules of a gas. Clausius went to the extent of introducing a statistical concept into the mechanical description of gases, namely the mean path traversed by a molecule between two collisions.

Clausius' work needs Statistics but did not use it. Maxwell made use of Statistics, not merely averages. He used the distribution function to make the proposal of the Kinetic Theory of gases quantitative and concrete. The distribution function gives the probability that a molecule has a given momentum vector. He took a box of volume $d^{3} \mathbf{p}$ about the momentum vector $\mathbf{p}$ in momentum space. The probability 
density, $f(\mathbf{p})$, is then the ratio of the number of molecules in the box divided by the total number of molecules in the system. Summing up over all such volumes in the momentum space $\int_{V} f(\mathbf{p}) d^{3} \mathbf{p}$ must be unity where $V$ is the volume of momentum space. This will then automatically lead to the velocity and energy distributions for the molecules. Now the average kinetic energy must be the thermal energy, which is a constant (called the Boltzmann constant, normally denoted by $k$ ) times the temperature. This explains the temperature. Mathematically, the temperature must be the weighted average of the kinetic energy of all the molecules with this distribution function, over an infinite momentum space. Writing the momentum space in spherical polar coordinates the average kinetic energy is then

$$
\langle K . E .\rangle=\frac{2 \pi}{m} \int_{0}^{\infty} f(\mathbf{p}) p^{4} d p:=\frac{3}{2} k T,
$$

having taken the average momentum of the molecules to be zero, where $m$ is the mass of the molecule. This gives the Maxwell momentum distribution

$$
f_{p}(\mathbf{p})=(2 \pi m k T)^{-3 / 2} e^{-p^{2}} / 2 \pi m k T \text {. }
$$

One can replace the momentum by the velocity, v, to obtain the Maxwell velocity distribution

$$
f_{v}(\mathbf{v})=\frac{4}{\sqrt{\pi}}\left(\frac{m}{2 k T}\right)^{3 / 2} v^{2} e^{-m v^{2} / 2 k T},
$$

and thence the Maxwell energy distribution

$$
f_{E}(E)=2 \sqrt{\frac{E}{\pi}}\left(\frac{1}{k T}\right)^{-3 / 2} e^{-E / k T} .
$$

For a given momentum vector, $\mathbf{p}, \mathbf{p} f_{p}(\mathbf{p}) d^{3} \mathbf{p}$ gives the probability of the momentum being $\mathbf{p}$, in the momentum space volume $d^{3} \mathbf{p}$. Integrating over the volume must give zero for the system to be at rest. Now, take the component of $\mathbf{p}$ along the vector area elements on the surface and integrate their product, p.dS , over the entire surface of the gas container. This gives the pressure, $P$ of the gas, which turns out to satisfy the ideal gas equation of state, $P V=N k T$, if we treat the molecules as point particles that do not interact, where $N$ is the number of molecules in the system. (Allowing for the volume and interactions of the molecules gives the van der Waals equation of state.)

This provides a demonstrable explanation of heat in terms of the mechanics of Newton. The difference between the classical picture and the kinetic picture can be tested by allowing a beam of hot gas to escape through an orifice in the container. Kinetic Theory predicts that the particles will spread out due to gravity in the up-down direction when they hit a screen, because there will be some with greater momentum that will go nearly straight and others with less momentum that will be pulled down more. The classical expectation would be that it should spread uniformly about the geometrical shadow of the hole on the screen. 
Does this theory explain the concept of entropy? Maxwell worried over this matter and finally came up with an explanation. He considered a gas consisting of two types of molecules in two joined boxes with a tiny hole in the partition between the two boxes. He now placed a microscopic demon (a name given by William Thomson) guarding the hole from one side of the hole. This demon would separate the molecules that cross the hole, so that over time there would be two pure gases in the two boxes, which violates the Second Law of Thermodynamics. Similarly, even for only one type of molecule, the demon could separate the fast from the slow molecules so that the temperatures of the two connected boxes would be different, again violating the Second Law. However, the work done by the demon in blocking and sending on the molecules has to be accounted for and so the demon does work to locally reduce the entropy, but increases its own by at least as much. The moral of this story is that in a system without such a demon, the molecules are stopped from separating into a hot and cold side, or into two types of molecules, by the improbability of the separation occurring spontaneously. How unlikely is it? Well, in a cubic centimetre of gas at normal temperature and pressure, the number of molecules is given by Avogadro's number, viz. $6 \times 10^{23}$. Consider air in a small cube of one centimetre side. It consists of nearly one part of oxygen to four parts of nitrogen, with some other gases mixed in. For our purposes, take it to be in precisely this ratio. Thus there are about $1.2 \times 10^{23}$ molecules of oxygen and $4.8 \times 10^{23}$ of nitrogen. The probability of the separation occurring spontaneously is, then about $51.8 \times 10^{-47}$, which is as good as zero.

Maxwell's great contribution to physical thinking here is the realization that the Second Law of Thermodynamics is statistical in nature. In a letter to Tait he said that he introduced the demon to show that the second law of thermodynamics is a statistical law which applies to all molecules and not to individual molecules. It is worth noting that the second law is arguably the most robust law in Nature. Even the first law breaks down in a Universe that changes with time, according to Einstein's theory of General Relativity, but the Second Law, being statistical, is robust. The larger the system the more accurate the assumption of things "running down". Contrariwise, the smaller the system, the less definite is the prediction. Another extremely important contribution is the use of the thought experiment, which was a standard technique of Einstein and used extensively to this day. We do not know of a prior use of a genuine thought experiment in the modern sense, namely one that cannot be performed in a laboratory. Thinking about Maxwell's demon helped Leo Szilard develop information theory and the Szilard engine in 1929. ${ }^{23}$ More recent developments of Maxwell's demon can be found in Refs. 24 and 25 .

The statistical methods of Maxwell were developed further by Boltzmann, using Hamilton's formulation of mechanics and forming the space constituted of both position and momentum, called phase space. In particular, he developed the MaxwellBoltzmann distribution that incorporates collisions into the theory. Though the formal definition of entropy as the natural logarithm of the number of phase space 
states available, relies on Boltzmann's formulation of Statistical Mechanics, its substance is already there in Maxwell's Kinetic Theory of Gases. These developments later led Max Planck to the postulate that the energy is absorbed and emitted by molecules in discrete quanta, which explained the observed behaviour of radiant energy. This led Einstein to postulate the existence of quanta of energy. Nowadays, the statistical thinking of Maxwell has been developed much further and is arguably the most crucial underlying paradigm.

\section{Electromagnetism}

When Maxwell started his work on electricity and magnetism, much had already been discovered. The concept of the electric and magnetic lines of force had been provided by Faraday. It is particularly easy to see for magnetism by plotting the direction of the needle of a small compass as one moves the compass along the lines of force about a bar magnet. They are seen still more easily by putting a bar magnet on a white sheet of paper and pouring iron filings on to the paper around the magnet. Since the filings behave like tiny iron needles, they perform the same job as the compass needle, but do so simultaneously, giving a picture of the field as a whole. Ampere and Faraday had already stated their Laws giving the magnetic effects of electric currents and the electric effects of moving magnets.

Maxwell formalized Faraday's more physical idea of "lines of force" as the field intensity, thereby providing the first clear concept of the electric and magnetic fields. Since the lines of force emanate from some source, they must have a starting point. Since we do find separate charges (electric monopoles) the electric lines of force need have no ends. However, since we do not see magnetic "charges" i.e. monopoles separately, the magnetic lines of force must have ends as well. Looking at the flux of lines of force emerging from some region we can use Gauss' divergence theorem to conclude laws for the electric and magnetic field intensities, $\mathbf{E}$ and $\mathbf{B}$,

$$
\nabla \cdot \mathbf{E}=\rho / \epsilon, \quad \nabla \cdot \mathbf{B}=0
$$

where $\nabla$. is the divergence operator, $\rho$ is the electric charge density and $\epsilon$ is the dielectric constant, which varies from material to material. It has a finite value for the vacuum, usually denoted by $\epsilon_{0}$, which will be relevant shortly. These laws are generally referred to as Gauss' laws.

Ampere's Law relates the magnetic flux across a loop of wire to the current flowing through it:

$$
\oint \mathbf{B} \cdot d \mathbf{S}=\mu I,
$$

where $d \mathbf{S}$ is the vector area element for the surface containing the loop of wire, $\mu$ is the magnetic permittivity which varies according to the material used for the surface in which the wire is embedded. For the vacuum it is $\mu_{0}$ and $I$ is the electric current. This can be written in differential (i.e. local) form instead of the integral 
form by using Stokes' theorem as

$$
\nabla \times \mathbf{B}=\mu \mathbf{j}
$$

where $\mathbf{j}$ is the current per unit length in a piece of the loop of wire.

Faraday's Law, that the electromotive force induced by a time-varying magnetic field is given by the rate of change of that field, can be stated mathematically as

$$
\nabla \times \mathbf{E}=-\frac{\partial \mathbf{B}}{\partial t}
$$

Equations (5), (7) (with a modification) and (8) are called the Maxwell equations. If all the laws were already in place and the magnetic effects of electric currents and the electric effects of moving magnets were already known, was there no genuine contribution by Maxwell? Did he simply compile all the results obtained by others in his treatise and receive the credit due to the rest? The answer is an emphatic "NO!" The clear formulation of the field concept is crucial to the modern understanding of Electromagnetism and other fields and provides the paradigm for modern Physics. Moreover, Maxwell immediately obtained many vital new insights and discovered new facts on their basis. Let us examine the contributions in more detail.

The idea of a field is there in embryonic form in Faraday's lines of force. However, it does not make clear the ramifications of that concept and the dramatic break it entails with the Newtonian view. While Newton had localized causes with corresponding local effects Maxwell provided the nonlocal field. Thus, while Newton had a problem with the "action at a distance" that his law of gravity entailed, Maxwell provided that action through the mediation of his all-pervasive field. Further, that field implied that all prior data everywhere would determine subsequent events everywhere and there would be no localized "causes" for any effects. Instead of action at a distance being odd, it is localized action that needs to be explained.

As if this contribution was not enough, the next one goes still deeper. Maxwell noted that there is an underlying symmetry between the electric and magnetic fields in the absence of sources. The Physics underlying it is that moving electric charges lead to magnetic fields and moving electric fields lead to magnetic effects, even where the charges are not present. This duality requires a fundamental unity in the two fields. This observation provides the paradigm for the search for unification of forces of Nature to this day. The further realization that where there is such a physical unification there will be some associated symmetry that is somehow broken, is equally significant - and is the reason Maxwell's contributions are being celebrated in the SDEA-II at the sesquicentennial of his presentation of the theory to the Royal Society. This, again, is an underlying paradigm for modern High Energy Physics and the unification attempts. The physical realization that it is the effect of each on the other that signals a unification is yet another vital insight that is used.

Maxwell immediately realized that Ampere's Law, Eq. (7), violated the required symmetry. Because of his deep conviction that symmetry will rule, he set about 
finding what was missing to complete the desired symmetry. The current density is just the rate of change of the charge density. There must, then also be the rate of change of the electric field which has to be inserted into that equation:

$$
\nabla \times \mathbf{B}=\mu \mathbf{J}+\epsilon \mu \frac{\partial \mathbf{E}}{\partial t} .
$$

He called the inserted term the "displacement current" and set about searching for it experimentally by looking for a deflection in a needle due to the varying electric field with no possibility for electric charges to flow. It is a tribute to his ingenuity and conviction that he found this term. Here was a case where he predicted that the prevailing theory was wrong and demonstrated an effect that was not consistent with that theory but fitted his theory. This, again, has become the paradigm for modern developments through Theoretical Physics, and indeed the driving force for doing laboratory experiments to-day.

For the next major contribution take the curl of Eq. (8), commute $\nabla$ with $\frac{\partial}{\partial t}$ and use Eqs. (9) and (5) and the vector operator identity that the curl of the curl of a vector is the gradient of the divergence of the vector minus the Laplacian acting on the vector to obtain the wave equation for the electric field with a source. Similarly, taking the curl of Eq. (9) and using the other three equations with the same identities, gives the wave equation with a source for the magnetic field. Hence there must be electromagnetic waves. In other words, these waves will be present even where there are no sources. The wave equation for the electromagnetic field obtained carries the speed of the wave in it. Identifying the speed by its square appearing as the coefficient of the Laplacian part of the wave operator, it turns out to be $1 / \sqrt{\epsilon \mu}$. Using the values for the vacuum one obtains a speed that is extremely close to the speed of light. Hence, not only did Maxwell discover that there would be electromagnetic waves but that light is an electromagnetic wave This discovery confirmed the wave theory of light and provided a complete testable explanation of such diverse phenomena as the dependence of the refractive index of a material on its dielectric constant and its magnetic permittivity, as well as providing the frequency of light in terms of its wavelength, of chromatic aberration and many other similar phenomena.

Potentials had been introduced to deal with the gravitational force in terms of the associated energy to simplify calculations by Lagrange and used by Laplace to discuss the gravitational force outside a gravitating body and by Poisson to extend it to the interior of gravitating bodies, yielding equations known by the names of their originators. The gravitational potential is a scalar function whose gradient is the negative of the gravitational force. Maxwell extended this to the electric and magnetic forces to obtain corresponding scalar potentials, $\phi$ and $U^{*}$. However, because the curl of a gradient is zero and so the divergence of a curl is zero, on account of Eq. (5), the magnetic field can be expressed as the curl of a vector potential, A, while the electric field cannot. Taking this fact to be fundamental, Maxwell used the vector potential for his further formulation. 
Notice that the addition of the gradient of an arbitrary scalar function of space and time to this vector potential would make no difference to the magnetic field. Hence the magnetic field is invariant under this transformation. However, to accommodate the displacement current Maxwell found that he had to re-define the electric potential, writing it as the sum of an electrostatic potential and the time derivative of the magnetic vector potential, so that

$$
\mathbf{E}=-\nabla \phi-\frac{\partial \mathbf{A}}{\partial t}
$$

Thus corresponding to the addition of the gradient to the magnetic vector potential there must be a subtraction of its time derivative in the electric potential. Mathematically, the electromagnetic field is invariant under the "gauge transformation" of the potentials,

$$
\mathbf{A} \rightarrow \tilde{\mathbf{A}}=\mathbf{A}+\nabla f(x, y, z, t), \quad \phi \rightarrow \tilde{\phi}=\phi-\partial f(x, y, z, t) / \partial t .
$$

To put the unified electromagnetic field in terms of potentials he used the four component generalization of the complex numbers called quaternions proposed by Hamilton. They are defined as $q=r+a i+b j+c k$, where $a, b, c, r \in \mathbb{R}, i^{2}=j^{2}=$ $k^{2}=-1$. He then noted that this new four component potential can be changed by adding the gradient of an arbitrary scalar function of space and time without making any difference to the physical electromagnetic field. This freedom of choice of the potential is called "gauge freedom". This was in some ways the most remarkable and significant of his many discoveries. The quarternions have often been considered for transcribing special relativity as the structure is remarkably similar to that of Minkowski space and essentially gives the same group structure as well.

From here Maxwell could have taken two short steps and arrived at the Special Theory of Relativity but was held back by his reverence for Newton's mechanics that had provided so many insights. One step involved in fully accepting the electromagnetic field as itself providing the medium in which light (or electromagnetic waves) move and thus dispensing with the old ether. Instead he spent much of his dwindling energy on trying to construct a mechanical model of the ether, which could support electromagnetic waves, to demonstrate that it could be done. The other was to pursue the fact that his theory gives a constant speed of light for all observers. Since the principle of special relativity was already taken for granted, no more was needed. (It may be mentioned that his Kinetic Theory of gases had also brought him to the verge of discovering the quantum of electromagnetic waves.)

Many physicists have placed Maxwell at par with Galilieo, Newton and Einstein as among the greatest ever, with no means of deciding who was the greater. In the case of the latter two, there was an "annus mirabiles" associated with each. For Maxwell there was no single such year but a whole stream of nearly equally fundamental developments flowed from him from about 1860 to about 1870, _ "anni mirabilis" or a "decade mirabiles". (Of course, for Einstein there was much more that he developed long after his "miracle year", notably his theory of general relativity and his application of that theory to cosmology.) Among those who consider 
Maxwell to be an "unsung hero" of Physics, was Abdus Salam (Nobel Laureate), who said it to one of us (AQ) personally, and his mentor Paul Adrian Maurice Dirac (Nobel Laureate) who he quoted. It is worth noting that Maxwell did not have the advantage of the modern tensor notation and developed all his ideas on the basis of his physical intuition and the cumbersome notation using separate symbols for each separate component of the vector fields and potentials. Seeing them written out in their full gory detail is an object lesson in the utility of modern tensor notation and a reminder of his genius, that he saw the essential simplicity inherent in the mess of his equations. When one sees the equations in the language of differential forms in four dimensions, with one definition of the field, one obvious set of identities and one working equation, one loses a sense of what he had to look at. He nevertheless set up the fundamental search for unification, with his theory of Electromagnetism, that was followed by Einstein's unification of space and time, based on Maxwell's unification of Electromagnetism and leading on to the gauge unification of the electromagnetic and weak nuclear forces and set the stage for the current attempts at the gauge unification of all forces of Nature.

Which of Maxwell's developments is taken as more important, the kinetic theory of gases or Electromagnetism, we leave to the readers to decide for themselves. The current consensus seems to be that the field concept and production of the model workable field theory is the more important. From the point of view of the conference on symmetries that would certainly be endorsed. After all, the key feature of Electromagnetism, the gauge freedom to choose the electromagnetic 4-vector potential, provides the basis of using symmetries for physical purposes. It plays a role through Noether's theorem and thence in the modern "gauge theories" of quantum field theory; be they Abelian or nonabelian. However, the importance of the second law of thermodynamics and its understanding provided by Maxwell may lead to that being regarded as more fundamental in later ages.

\section{Conclusion}

The contribution of Maxwell that we celebrate in this paper relates to Electromagnetism, which is regarded as his greatest achievement, not only as the introduction of the field concept but also as the simplest and most elegant example of a field theory. While much of the technological use of electricity does not require his field theory, there is so much that does, such as its use in wave guides and hence in fibre optics, its use in the theory of refraction and its use for the propagation of radio and television waves, that one wonders how much of the modern world would have been possible without it. Although it is because of its use that its significance may be appreciated by everybody, it is because of its development of the human understanding of the world we live in that it may be considered to have had the most dramatic impact. It provides the basic language of the Physics of today and lies at the root of Albert Einstein's special theory of relativity. One may recall that the original paper in relativity was entitled On the electrodynamics of moving 
bodies. ${ }^{26}$ It has generally been assumed that despite having introduced the field concept which obviates the need for a luminiferous aether, Maxwell continued to believe in it. However, it has been pointed out that he may have given it as a sop to the conservatism of his colleagues of the time, to be dispensed with at a future time. Of course, we will now never know which was his true belief.

We have also mentioned numerous other contributions of Maxwell, while still missing many others. One that we did dwell on was his development of the Kinetic Theory of gases that lies at the base of the other major method of modern Physics, namely Statistical Mechanics. It is worth mentioning one aspect of the introduction of his "demon". To the best of our knowledge this is the first real thought experiment used in Physics. Previous such experiments were merely idealizations of experiments that could actually have been performed, such as Galileo's smoothly sailing boat to illustrate his principle of the relativity of uniform linear motion. Maxwell's demon is not such a thought experiment. It makes a point of principle that comes out loud and clear by considering an impossible experiment. Such "experiments" have now become the mainstay of theoretical physical reasoning.

Maxwell died very young at the age of 48. It is interesting to speculate how much he might have achieved had he lived to a riper age with the good health that so many later physicists have enjoyed. It is certainly not fanciful to speculate on his discovering Special Relativity, which is a direct consequence of his theory that we are celebrating. For that matter, Einstein formulated his General Relativity as a tensor field theory; a direct generalization of the Maxwell vector field theory. Again, it is certainly not pushing credulity to suppose that he would soon have developed Statistical Mechanics. With his interest in the theory of radiation, he may even have arrived at Planck's quantum of energy emitted or absorbed.

He seems to have been a twentieth century man trapped in the nineteenth century, as the following excerpts form his letters show. They enhance the scientist, the teacher, the man with his knowledge, his beliefs and his wittiness.

Letter to Professor WG Adams, Natural Science Tripos, 3rd December 1873: ${ }^{10}$

I do not approve of the plan of a physical society considered as an instrument for the improvement of natural knowledge. If it is to publish papers on physical subjects which would not find their place in the transactions of existing societies, or in scientific journals, I think the progress towards dissolution will be very rapid. (omissis) For the evolution of science by societies the main requisite is the perfect freedom of communication between each member and any one of the others who may act as a reagent.

Letter to Rev. CJ Ellicott, Lord Bishop of Gloucester and Bristol, 11 Scroope Terrace, Cambridge, 22nd November 1876: ${ }^{10}$

But I should be very sorry if an interpretation founded on a most conjectural scientific hypotheses were to get fastened to the text in Genesis, even if by so doing it got rid of the old statement of the commentators which has long ceased to be intelligible. The rate of change of scientific hypothesis is naturally much more rapid 
than that of Biblical interpretations, so that if an interpretation is founded on such an hypothesis, it may help to keep the hypothesis above ground long after it ought to be buried and forgotten. At the same time I think that each individual man should do all he can to impress his own mind with the extent, the order, and the unity of the universe, and should carry these ideas with him as he reads such passages as the 1st Chap. of the Ep. to Colossians (see Lightfoot on Colossians, p. 182), just as enlarged conceptions of the extent and unity of the world of life may be of service to us in reading Psalm viii.: Heb. ii. 6, etc.

Letter to Mrs Maxwell, Trinity College, Cambridge, 3rd January 1870: ${ }^{10}$

There is a tradition in Trinity that when I was here I discovered a method of throwing a cat so as not to light on its feet, and that I used to throw cats out of windows. I had to explain that the proper object of research was to find how quick the cat would turn round, and that the proper method was to let the cat drop on a table or bed from about two inches, and that even then the cat lights on her feet.

\section{Acknowledgements}

We thank the referees for useful comments and suggestions. DPM thanks the National Research Foundation, Pretoria, South Africa, for financial support.

\section{References}

1. JC Maxwell, "A dynamical theory of the electromagnetic field", Philos. Trans. Roy. Soc. Lond. CLV (1865) 459-512.

2. WD Niven, The Scientific Papers of James Clerk Maxwell Vols. 1 \& 2 (Cambridge University Press, Cambridge, 1980).

3. I Tolstoy, James Clerk Maxwell (Canongate, Edinburgh, 1981).

4. James Clerk Maxwell Commemorative Booklet produced by the James Clerk Maxwell Foundation on the occasion of the Fourth International Congress on Industrial and Applied Mathematics coming to Edinburgh in July 1999.

5. I James, Remarkable Physicists: From Galileo to Yukawa (Cambridge University Press, Cambridge, 2004).

6. B Mahon, The Man who Changed Everything: The Life of James Clerk Maxwell (John Wiley and Sons, Chichester, 2004).

7. JC Maxwell, "On the description of oval curves and those having a plurality of foci", Proc. Roy. Soc. Edinb. II April 1851 (1844-1850) 89-91.

8. JC Maxwell, "On the theory of rolling curves", Trans. Roy. Soc. Edinb. XVI (1849) 519-540.

9. JC Maxwell, "On the equilibrium of elastic solids", Trans. Roy. Soc. Edinb. XX (1853) $87-120$.

10. L Campbell and W Garnett. Life of James Clerk Maxwell, MacMillan and Co., London, 1882.

11. JC Maxwell, "Experiments on colour, as perceived by the eye, with remarks on colour blindness", Trans. Roy. Soc. Edinb. XXI (1857) 275-289.

12. Class for Physics of the Royal Swedish Academy of Sciences, Scientific Background on the Nobel Prize in Physics 2014. Efficient blue light-emmitting dioes leading to bright and energy-saving white hight sources. Nobelprizw.org.2014. 
13. JC Maxwell, "On Faraday's lines of force", Trans. Camb. Philos. Soc. X (1864) 27-83.

14. JC Maxwell, "On the theory of compound colours and the relations of the colours of the spectrum", Philos. Trans. Roy. Soc. Lond. 150 (1861) 57-84.

15. WM Smart. John Couch Adams and the Discovery of Neptune, Nature 158, 648-652, 1946.

16. JC Maxwell, "On the stability of the motion of Saturn's rings", Monthly Notices of the Roy. Astronom. Soc. 10 (1859) 297-384.

17. JC Maxwell, "Illustrations of the dynamical theory of gases I: On the motion and collision of perfectly elastic spheres", Philos. Mag. XIX (1860) 19-32; II "On the process of diffusion of two or more kinds of moving particles among one another", 33-37.

18. JC Maxwell, "On the dynamical theory of gases", Phil. Trans. Roy. Soc. Lond. CLVII (1868) 49-88.

19. JC Maxwell, "On hills and dales", Philos. Mag. XL (1870) 421-426.

20. JC Maxwell, Theory of heat (Text-books of Science, London, 1870).

21. JC Maxwell (ed.), The electrical researches of the Honourable Henry Cavendish FRS written between 1771 and 1781, edited from the original manuscripts in the possession of the Duke of Devonshire, $K G$ (Cambridge, 1879).

22. JC Maxwell, "On Boltzmann's theorem on the average distribution of energy in a system of material points", Trans. Camb. Philos. Soc. XII (1879) 547-570.

23. M Klein, Maxwell, his Demon, and the Second Law of Thermodynamics, American Scientist 58 84-97, 1970.

24. EE Daub, Maxwell's Demon, Studies in History and Philosophy of Science, Part A. Volume 1, Issue 3, 213-227, 1970.

25. H Leff and AF Rex (Eds), Maxwell's Demon 2: Entropy, Classical and Quantum Information, Computing, Institute of Physics, London, 2003.

26. A Einstein, "Zur elektrodynamik bewegter körper", Annalen der Physik 17 (1905) 891; English translation "On the electrodynamics of moving bodies" by M. Saha University of Calcutta 1920; and later by G.B. Jeffery and W. Perrett, Methuen and Co. Ltd. 1923. 